\title{
Cervical HSIL Involving the Endometrium and Adenomyosis: A Case Report and Literature Review
}

\author{
Wenwen Wang and Feng Zhou \\ Department of Pathology, Women's Hospital, School of Medicine, Zhejiang University, Hangzhou, Zhejiang Province, China
}

\begin{abstract}
Cervical squamous cell carcinoma (SCC) spreading superficially to the inner surface of the uterus and replacing the endometrial lining cells with carcinoma cells is called superficial spreading SCC. We, hereby report a 53-year postmenopausal woman who had a history of cervical SCC in situ/high grade squamous intraepithelial lesion (HSIL). Ultrasonography revealed an enlarged uterus measuring $9.0 \times 6.5 \times 5.0 \mathrm{~cm}$ with dilatation of the uterine cavity and the cervical canal. Subsequently, she underwent total abdominal hysterectomy (TAH) and bilateral salpingo-ophorectomy with a diagnosis of cervical HSIL involving the endometrium and adenomyosis. This a very rare phenomenon, as the endometrial involvement may be caused by direct extension of the cervical lesion, and occur mainly in postmenopausal women, and should be deligently searched during pathological examination.
\end{abstract}

Key Words: HSIL, Cervix, Endometrium, Adenomyosis.

How to cite this article: Wang W, Zhou F. Cervical HSIL Involving the Endometrium and Adenomyosis: A Case Report and Literature Review. J Coll Physicians Surg Pak 2021; 31(03):337-339.

\section{INTRODUCTION}

Cervical squamous cell carcinoma (SCC) is one of the common malignancies of the female genital tract. Cervical SCC, spreading superficially to the inner surface of the uterus and replacing the endometrium with carcinoma cells, is called superficial spreading SCC. ${ }^{1}$ Cervical SCC in situ/high grade squamous intraepithelial lesion (HSIL) spreading to the endometrium is rare. ${ }^{2-6}$ Here, we present a case of cervical HSIL spreading to the endometrium and adenomyosis.

\section{CASE REPORT}

A 53-year woman was referred to the Oncology Clinic for an abnormal Papanicolaou (PAP) smear. The patient had been menopausal for the past three years.

Physical examination showed atrophic cervix with no contact bleeding and no tenderness on palpation of the bilateral adnexe. Ultrasonography revealed an enlarged uterus measuring $9.0 \times 6.5 \times 5.0 \mathrm{~cm}$ with dilatation of the uterine cavity and the cervicalcanal.

Correspondence to: Dr. Feng Zhou, Department of Pathology, Women's Hospital, School of Medicine, Zhejiang University, Hangzhou, Zhejiang Province, China

E-mail: fungchew@zju.edu.cn

Received: March 07, 2019; Revised: October 23, 2019;

Accepted: December 09, 2019

DOI: https://doi.org/10.29271/jcpsp.2021.03.337
Her serum carcinoembryonic antigen (CEA), cancer antigen (CA)-125, and CA19-9 levels were normal. With the cervical HSIL confirmed by punch biopsy, a loop electrosurgical excision procedure (LEEP) was performed. Histologic evaluation of the specimen showed a cervical HSIL with positive endocervical margin. The patient subsequently underwent total abdominal hysterectomy (TAH) and bilateral salpingo-ophorectomy.

On macroscopic examination, the uterus was $8.0 \times 5.0 \times 5.0$ $\mathrm{cm}$. The cervix was thin. The uterine cavity was filled with pus. The inner surface of the cavity showed a superficial yellowish exudate, extending from the endocervical canal. Both fallopian tubes and ovaries were grossly unremarkable.

Microscopic examination of the cervix disclosed atypical squamous cell proliferation confined to the epithelial layer. These atypical cells exhibited high nuclear to cytoplasmic ratio, rounded nuclei with distinct nucleoli and high mitotic activity (Figure 1A). Cervical stromal invasion was not observed. The endometrium was replaced by sheets of atypical squamous cells with marked pleomorphism and bizarreforms without invasion of the underlying myometrium (Figure 1B). This endometrial lesion was directly contiguous to the cervical lesion. Endometrial glands and stroma, located more than $5 \mathrm{~mm}$ deep from the endometrial basal layer, were also noted. Some endometrial glands within this adenomyotic focus were also replaced by atypical squamous cells (Figure $1 C \& D$ ).

Immunohistochemically, the atypical squamous cells of the cervix and endometrium were strongly positive for $p 16$ and p63, and a high Ki67 labelling index was noted. 
Thus, the final diagnosis was cervical HSIL, involving the endometrium and adenomyosis. The patient did not receive additional therapy and was symptom-free six months after surgery.
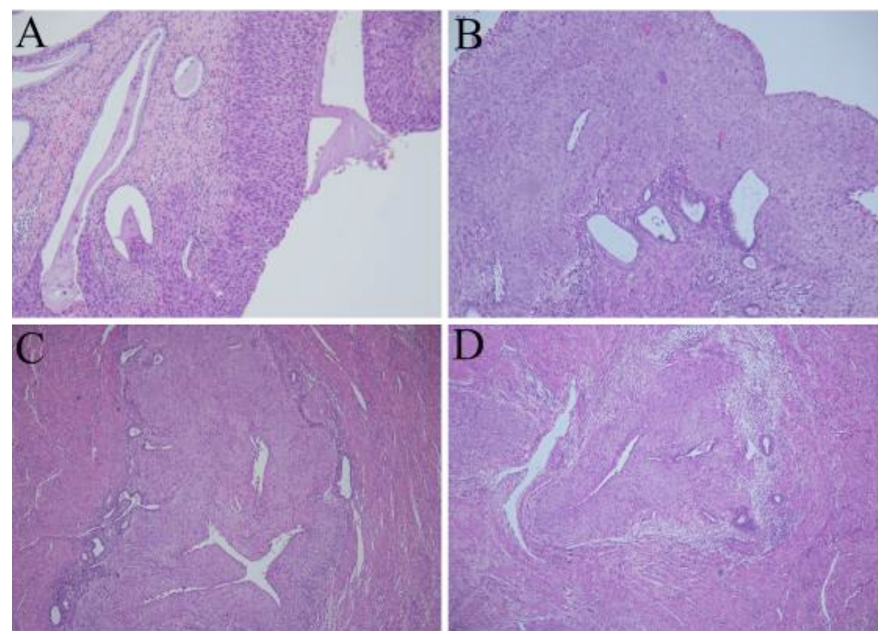

Figure 1: Histological findings (H\&E): HSIL of the cervix (A 10x); HSIL covering the normal endometrial glands (B 10X); HSIL involving the adenomyosis (C, D 5x).

\section{DISCUSSION}

Cervical HSIL spreading to the endometrium is rare and has been primarily limited to case series. ${ }^{2-6}$ Adenomyosis is commonly seen with endometrial adenocarcinoma and may be involved by the adenocarcinoma. However, adenomyosis involvement by cervical HSIL has not been reported. In this study, we describe a rare case of superficially spreading HISL of the cervix, involving both the endometrium and adenomyosis.

This rare occurrence should be distinguished from ichthyosis uteri, which is also a rare condition, in which, the entire surface of the endometrium is replaced by stratified squamous epithelium. ${ }^{7}$ In this case, the tumor cells had obvious dysplasia and were strongly positive for p16 having a high Ki67 index, a typical feature of endometrial HSIL.

To our knowledge, no cases with almost similar spreading features were reported where the involvement reached the adenomyosis. Adenomyosis involved by HSIL can easily be misdiagnosed as invasive SCC. However, normal endometrial glands can be seen around the involved face of adenomyosis. Nostromal reactions such as oedema, inflammationand desmoplasia were observed, which are common in invasive carcinoma. In addition, a defined basement membrane, separating the lesions from the stroma, was also noted.

It is important to exclude primary endometrial squamous cell carcinoma (PESCC). PESCC should have no evidence of a co-existing endometrial adenocarcinoma or primary cervical SCC; no connection between the endometrial tumor and cervical squamous epithelium; no connection between any cervical SCC in situ and endometrial neoplasm. ${ }^{8,9}$ Our case did not satisfy these criteria.

The presence of endometrial lesion, associated with HSIL of the cervix, is suggestive of a superficial spread. However, the mechanism of the superficial spread of HSIL is not well understood. The characteristics of HSIL superficial spread includes a radical growth phase, which may precede vertical or invasive neoplastic growth. One study suggested that HSIL spreads horizontally along the basement membrane by mechanical displacement of the columnar epithelium. ${ }^{2}$

Normally, cervical HSIL is found in young women. However, the average age of reported spreading HSIL cases is nearly 60 years, and all were menopausal or post-menopausal. ${ }^{2-6}$ The patient in this case was similar to previously-reported cases based on age incidence (53 years) and menopausal status (three years post-menopausal). Thus, we hypothesise that atrophy of the endometrium and lack of periodic abscission may lead to this rare phenomenon.

In conclusion, cervical HSIL spreading to the endometrium and involving face of adenomyosis is a very rare phenomenon. Endometrial lesions may be caused by direct extension of the cervical lesion and may occur mainly in postmenopausal women, which should be thoroughly looked for during pathologicalexamination.

\section{ETHICALAPPROVAL:}

This study was approved by the hospital ethics committee.

\section{PATIENT'S CONSENT:}

The patient provided informed consent to participate in this study; and for publication of the data and accompanying images.

\section{CONFLICT OF INTEREST:}

The authors declared no conflict of interest.

\section{AUTHORS' CONTRIBUTIONS:}

WW: Took part in drafting, revising or critically reviewing the article.

FZ: Gave final approval of the version to be published.

All authors made a significant contribution to the work reported, whether that is in the conception, study design, execution, acquisition of data, analysis and interpretation, or in all these areas.

\section{REFERENCES}

1. Ishida $M$, Okabe $H$. Superficial spreading squamous cell carcinoma of the uterine cervix involving the endometrium: Report of two cases with emphasis on the likely molecular mechanism. Oncol Lett 2013; 5(1): 31-4. doi: 10.3892/ ol.2012.953.

2. Ferenczy A, Richart RM, Okagaki T. Endometrial involvement by cervical carcinoma in stitu. Am J Obstet Gynecol 1971; 110(4):590-2. doi.org/10.1016/00029378(71)90706-X.

3. Daniele E, Perino A, Catinella E. Superficial endometrial involvement by cervical intraepithelial neoplasia detected by intrauterine cytology: A case report. Acta Cytol 1985; 29(3):411-3.

4. Gupta S, Gupta IM, Tiwari PV. In-situ carcinoma of the 
uterine cervix showing superficial endometrial spread. Acta Obstet Gynecol Scand 1979; 58(5):507-8. doi: 10.3109/ 00016347909154079.

5. Anthuenis J, Baekelandt J, Bourgain C, De Rop C. Squamous cell carcinoma in situ lining the uterine cavity. Eur J Gynaecol Oncol 2016; 37(1): 135-8.

6. Yang SW, Kim WY, Cho SH, Yoon SH, Lim JY, Leet SJ. Multifocal microinvasive squamous cell carcinoma with extensive spread of squamous cell carcinoma in situ (CIS) into the uterine corpus, vagina, and left salpinx diagnosed five years after conisation of cervical CIS. Eur J Gynaecol Oncol 2014; 35(5):600-3.
7. Jain M, Kashyap A, Biswas R. Primary endometrial squamous cell carcinoma in-situ with extensive icthyosis uteri: A rare case report. J Clin Diagn Res 2017; 11(8): ED13-4. doi: 10.7860/JCDR/2017/29967.10384.

8. Farhane FZ, Alami Z, Bouhafa T, Elmazghi A, Hassouni K. Primary squamous cell carcinoma of endometrium: case report and literature review. Pan Afr Med J 2018; 30:208. doi: 10.11604/pamj.2018.30.8.8983.

9. Zhang C, Zhang H, Yang L, Wang Y, Li X, Guo J, et al. Primary squamous cell carcinoma of the endometrium in a woman of perimenopausal age: A case report. Medicine (Baltimore) 2018; 97(48):e13418. doi: 10.1097/MD.000 0000000013418. 